\title{
Bifurcations in a two-mass model of the vocal folds
}

\author{
Jorge C. Lucero
}

Department of Computer Science, University of Brasília, Brasília DF 70910-900, Brazil

(Received 23 October 1995)

Keywords: Bifurcation, Two-mass model, Vocal folds, Phonation, Vocal register PACS number: 43. 70. Aj, 43 70. Bk

\section{Introduction}

The dynamics of the vocal fold oscillation was analyzed in a previous work ${ }^{1)}$ using a simplified version of the two-mass model. ${ }^{2}$ Three equilibrium positions were found, and a bifurcation diagram was derived using a coupling stiffness coefficient and the lung pressure as control parameters. It was shown that the oscillation region can be divided into two subregions, which correspond to oscillation around different equilibrium positions. This result was a novel finding for the oscillation dynamics, and has been confirmed in later works. ${ }^{3)}$ However, no interpretation of its meaning in terms of phonation has been offered yet. In this letter we will attempt an interpretation, showing that each subregion could be related to a different vocal register, one for the chest and the other for the falsetto.

The present analysis has also the purpose of updating the previous results where the original equations of the two-mass model were used, incorporating improvements to the aerodynamic equations of the two-mass model suggested recently in the literature. ${ }^{3,4)}$

\section{Two-mass model equations}

We neglect nonlinearities in the mechanical properties of the tissues, and the influence of the vocal tract and subglottal system. It has been shown that these simplifications do not alter significantly the basic dynamics of the oscillation. ${ }^{1,3,5)}$ The glottal aerodynamical equations are modified from the original equations of the model, ${ }^{2}$ assuming Bernoulli flow below the glottal constriction, and a free downstream jet above it. ${ }^{3,4)}$ With the above assumptions, the equations of motion are

$$
\left\{\begin{array}{l}
m_{1} \ddot{x}_{1}+r_{1} \dot{x}_{1}+s_{1}+k_{\mathrm{c}}\left(x_{1}-x_{2}\right)=F_{1} \\
m_{2} \ddot{x}_{2}+r_{2} \dot{x}_{2}+s_{2}+k_{\mathrm{c}}\left(x_{2}-x_{1}\right)=0
\end{array}\right.
$$

where $x_{i}(i=1,2)$ is the displacement of mass $m_{i}$ from its rest position, $r_{i}$ is the damping coefficient, $k_{\mathrm{c}}$ is the coupling stiffness, $s_{i}$ is the elastic restoring force given by

$$
s_{i}=\left\{\begin{array}{l}
k_{i} x_{i}, \text { for } x_{i}>-x_{0} \\
k_{i} x_{i}+h_{i}\left(x_{i}+x_{0}\right), \text { for } x_{i} \leq-x_{0}
\end{array} \quad(i=1,2)\right.
$$

where $k_{i}$ is the stiffness coefficient, $h_{i}$ is the extra stiffness coefficient introduced by the collision between the opposite folds, $x_{0}$ is the mass displacement where collision occurs; and $F_{1}$ is the aerodynamic force on mass $m_{1}$, given by

$$
F_{1}=\left\{\begin{array}{l}
d_{1} l_{\mathrm{g}} P_{\mathrm{s}}\left[1-\frac{\left(x_{2}+x_{0}\right)^{2}}{\left(x_{1}+x_{0}\right)^{2}}\right], \text { for } x_{1} \geq x_{2}>-x_{0} \\
0, \text { otherwise }
\end{array}\right.
$$

where $d_{1}$ is the width of mass $m_{1}, l_{\mathrm{g}}$ is its length, and $P_{\mathrm{s}}$ is the subglottal pressure.

\section{Bifurcation diagram}

As shown in the previous work, ${ }^{1)}$ the equilibrium positions appear only in the open glottis condition $x_{1}>-x_{0}, x_{2}>-x_{0}$. Setting the time derivatives to zero in the equations of motion (1), we obtain two solutions for the equilibrium positions: the rest position $x_{1}=0, x_{2}=0$, and a second position given by the simultaneous equations

$$
\begin{gathered}
x_{2}=\alpha x_{1} \\
k_{1}\left[1+\alpha \frac{k_{2}}{k_{1}}\right]\left(x_{1}+x_{0}\right)^{2}-d_{1} l_{\mathrm{g}} P_{\mathrm{s}}\left(1-\alpha^{2}\right)\left(x_{1}+x_{0}\right) \\
-d_{1} l_{\mathrm{g}} P_{\mathrm{s}} x_{0}(1-\alpha)^{2}=0
\end{gathered}
$$

under the condition $x_{1} \geq x_{2}$. In Eq. (4), $\alpha$ is the coupling coefficient

$$
\alpha=\frac{k_{\mathrm{c}}}{k_{2}+k_{\mathrm{c}}}
$$

Note that Eq. (5) has two solutions for $x_{1}$; however, only one of them belongs to the open glottis condition. Also, the second equilibrium position requires a rectangular or convergent glottis $\left(x_{1} \geq x_{2}\right)$.

The two equilibrium positions may become coincident at $x_{1}=0, x_{2}=0$. Introducing these values in Eq. (5), we obtain for the subglottal pressure

$$
P_{\mathrm{s}}=\frac{k_{1} x_{0}\left(1+\alpha k_{2} / k_{1}\right)}{2 d_{1} l_{\mathrm{g}}(1-\alpha)}
$$

For a subglottal pressure lower than this value, the only equilibrium position is the rest position. At and above it, both equilibrium positions exist.

In our previous analysis, ${ }^{1)}$ three rquilibrium positions were found. The third one was associated to an additional pressure drop at the glottal entry due to the formation of a vena contracta in the airstream, which had not been included in the present analysis. This equilibrium position was judged as not significant to 
the overall dynamics; its absence here would confirm that conclusion. Also, the second equilibrium position was found to exist in both convergent and divergent glottis. The present assumption of a jet above the glottal construction eliminates this position in the divergent case.

The stability of each equilibrium position may be next determined linearizing the equations of motion around each equilibrium position, and the examining the roots of the corresponding characteristic equation.

We consider the standard configuration given by the parameters $l_{\mathrm{g}}=1.4 \mathrm{~cm}, d_{1}=0.25 \mathrm{~cm}, x_{0}=0.02 \mathrm{~cm}, m_{1}=$ $0.125 \mathrm{~g}, \quad m_{2}=0.025 \mathrm{~g}, \quad k_{1}=80,000 \mathrm{dyn} / \mathrm{cm}, \quad k_{2}=8,000$ $\mathrm{dyn} / \mathrm{cm}, r_{1}=20 \mathrm{dyn} \mathrm{s} / \mathrm{cm}$.

The results are illustrated by the bifurcation diagram in Fig. 1. Curves BC and BE are Hopf bifurcations for the rest position and the second equilibrium position, respectively, and delimit the oscillation region EBC. Curve AD is given by Eq. (7), and indicates the coincidence of both equilibrium positions. Below the curve,

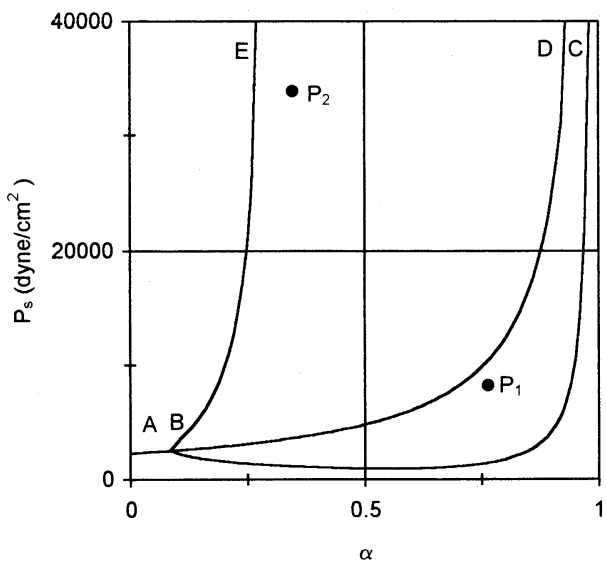

Fig. 1 Bifurcation diagram of the two-mass model.

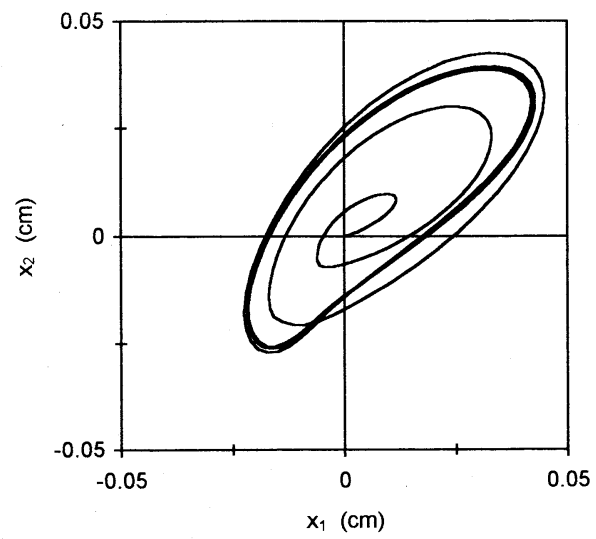

Fig. 2 State space plot for $\alpha=0.75$ and $P_{\mathrm{s}}=$ 8,000 dyn $/ \mathrm{cm}^{2}$ (point $P_{1}$ in Fig. 1).

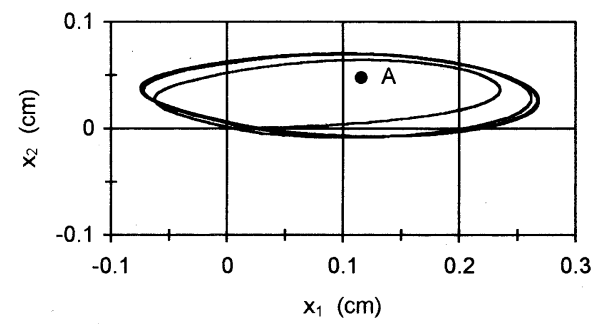

Fig. 3 State space plot for $\alpha=0.3$ and $P_{\mathrm{s}}=$ 35,000 dyn $/ \mathrm{cm}^{2}$ (point $\mathrm{P}_{2}$ in Fig. 1). Point A is the location of the second equilibrium position.

the only equilibrium position is the rest position; whereas at and above it, both equilibrium position exist. As discussed in the previous analysis, ${ }^{1)}$ this curve has the effect of exchanging the equilibrium positions involved in the two Hopf bifurcations.

Two state space plots are shown in Figs. 2 and 3, which corresponds to points $P_{1}$ and $P_{2}$ in Fig. 1, respectively. Note that in the case of Fig. 2, the oscillation takes place around the rest position; whereas in the case of Fig. 3, it does it around the second equilibrium position (point A). This may be explained by the bifurcation diagram noting that point $P_{1}$ is below curve $\mathrm{AD}$, in the region where the only equilibrium position is the rest position. Point $P_{2}$ is above curve AD, near the Hopf bifurcation involving the second equilibrium position.

\section{Discussion}

Figure 1 shows that the oscillation region EBC is divided by curve $\mathrm{AD}$ into two subregions, in which the oscillation occurs around different equilibrium positions: the rest position in subregion DBC, which is associated to a rectangular glottal $\left(x_{1}=x_{2}\right)$, and the second equilibrium position in subregion EBD, which is associated to a convergent glottal shape $\left(x_{1}>x_{2}\right)$. We may compare this analytical result with previous exparimental observations of the oscillation, where the same glottal shapes have been observed during oscillation at the chest and falsetto registers, respectively. ${ }^{6}$ This suggests the possibility of relating subregion DBC to vocal fold oscillation in the chest register, and subregion EBD to the falsetto register.

Further analytical efforts on more sophisticated models of the vocal folds are desirable as a next step to confirm this conclusion, which would explain vocal registers as a consequence of vocal fold oscillation around different equilibrium positions.

\section{Acknowledgements}

I am grateful to Dr. Wagner Teixeira for his interest and support. This research was funded by grant \#300587/93-7 of the National Council for Scientific and Technological Development of Brazil. 


\section{References}

1) J. C. Lucero, "Two-mass model of the vocal folds : Equilibria, bifurcations, and oscillation region," J. Acoust. Soc. Am. 94, 3104-3111 (1993).

2) K. Ishizaka and J. L. Flanagan, "Synthesis of voiced sounds from a two-mass model of the vocal folds," Bell Syst. Tech. J. 51, 1233-1268 (1972).

3) I. Steinecke and H. Herzel, "Bifurcations in an asymmetric vocal-fold model,” J. Acoust. Soc. Am. 97,
1874-1884 (1995).

4) B. H. Story and I. R. Titze, "Voice simulation with a body-cover model of the vocal folds," J. Acoust. Soc. Am. 97, 1249-1260 (1995).

5) I. R. Titze, "The physics of small-amplitude oscillation of the vocal folds," J. Acoust. Soc. Am. 83, 15361552 (1988).

6) I. R. Titze, Principles of Voice Production (PrenticeHall, Englewood Cliffs, 1994), pp. 259-262. 